\title{
Promoção da saúde e redução de vulnerabilidades por meio da prática da atividade física
}

\section{Promoting health and reducing vulnerability through physical activity}

\author{
Isabela Souza Cruvinel Borges ${ }^{1}$ (D) $\mid$ isabelascborges@gmail.com \\ Ana Clara Naves Vieira' (D) anaclaranv@hotmail.com \\ Igor Silva Campos ${ }^{1}$ (D) igor06silva@hotmail.com \\ Juliana Kanaan Machado' (D) julianakanaanm@gmail.com \\ Gustavo Antonio Raimondi' (D) gustavo.raimondi@gmail.com
}

\section{RESUMO}

Introdução: Diante da pandemia da Covid-19, os(as) estudantes de Medicina tornaram-se ainda mais fragilizados(as) e vulnerabilizados(as) pelo contexto de isolamento social, uma vez que tiveram suas rotinas alteradas. Assim, a Associação Atlética Acadêmica Marcel Resende Davi (AAAMRD), visando cumprir com a sua responsabilidade social de promoção da saúde, buscou incentivar a prática desportiva ao valorizar esse hábito em prol do bem-estar físico e mental dos(as) estudantes. Desse modo, este artigo objetiva relatar a experiência da atlética com a realização de um desafio esportivo virtual entre suas equipes.

Relato de experiência: O desafio foi elaborado e executado remotamente para 112 atletas de 14 equipes esportivas. Nos grupos de WhatsApp ${ }^{\oplus}$, particulares de cada time, os(as) atletas deveriam enviar mídias dos exercícios físicos realizados e contabilizá-los em uma lista. Ressalta-se que foram disponibilizados treinos nas redes sociais da atlética, em parceria com profissional capacitado, como forma de proporcionar treinos seguros. Ao final das 11 semanas de desafio, as três melhores equipes foram premiadas. Aplicou-se também um formulário de feedback para verificar a efetividade da ação.

Discussão: A tecnologia, importante aliada na organização do desafio, permitiu que a promoção da saúde ocorresse remotamente, respeitando o distanciamento social. Ao estimular a constância de treinos, a atlética contribuiu para a manutenção de parte da rotina dos(as) graduandos(as), prejudicada pelo contexto pandêmico. Além disso, o espírito de coletividade e a interação entre os(as) atletas dentro dos grupos contribuíram para que esse distanciamento não fosse total, formando uma rede de apoio ao compartilharem também seus medos, inseguranças e saudades.

Conclusão: Os resultados alcançados com o desafio e os feedbacks positivos confirmaram a efetividade da promoção da saúde proposta pela AAAMRD. Ademais, em conformidade com as Diretrizes Curriculares Nacionais e apesar do momento adverso, a instituição mostrou-se presente na formação pessoal e profissional dos(as) estudantes.

Palavras-chave: Promoção da Saúde; Responsabilidade Social; Educação Médica; Pandemia; Populações Vulneráveis.

\begin{abstract}
Introduction: In the face of the COVID-19 pandemic, medical students have become even more fragile and vulnerable due to social isolation, since their routines have been altered. Thus, the Marcel Resende Davi Academic Athletic Association (AAAMRD), fulfilling its social responsibility to promote health, sought to encourage the practice of sports, highlighting the benefit of this habit for students' physical and mental well-being. This article aims to report the institution's experience of organising a virtual sports challenge among its teams.

Experience report: The challenge was designed and executed remotely for 112 athletes from 14 sports teams. In Whatsapp ${ }^{\circledR}$ groups, private to each team, athletes were asked to send media of the physical exercises they performed and count them in a list. It is noteworthy that training was made available on the AAAMRD social networks, in partnership with a trained professional, as a way to provide safe training. At the end of the 11-week challenge, the top three teams were awarded. A feedback form was also applied to verify the effectiveness of the action.
\end{abstract}

Discussion: Technology, an important ally in organizing the challenge, allowed health promotion to take place remotely, respecting social distance. By encouraging constant training, the AAAMRD helped maintain part of the undergraduate students' routine, hampered by the pandemic. Moreover, the collective spirit and interaction between athletes, within their respective groups, corroborated to reduce the distance between them, forming a support network in which they could also share their fears and insecurities.

Conclusion: The results achieved through the challenge and the positive feedback confirmed the effectiveness of the health promotion proposed by AAAMRD. Furthermore, in accordance with the Brazilian National Guidelines for Undergraduate Medical Education and, despite the adverse moment, the institution remained present in the students' personal and professional development.

Keywords: Health Promotion; Social Responsibility; Medical Education; Pandemic; Vulnerable Populations.

${ }^{1}$ Universidade Federal de Uberlândia, Uberlândia, Minas Gerais, Brasil.

Editora-chefe: Daniela Chiesa

Editor associado: Roberto Zonato Esteves

Recebido em 30/08/20; Aceito em 15/12/20.

Avaliado pelo processo de double blind review. 


\section{INTRODUÇÃO}

A graduação em Medicina consiste em uma fase extenuante na vida da maioria dos(as) estudantes que, ao lidarem com a extensa "grade horária" e com a falta de tempo para estudar e participar das atividades universitárias, adotam um estilo de vida pouco saudável que afeta a própria saúde'. Atualmente, existe uma maior preocupação com a qualidade de vida desses(as) estudantes, o que envolve aspectos físicos, psicológicos e sociais ${ }^{1}$. Considerados(as) vulneráveis ao adoecimento por estarem expostos(as) a potenciais danos, em virtude da delicadeza atrelada às suas realidades, esses(as) alunos(as) constituem um grupo de risco, sendo os(as) mais afetados(as) por transtornos físicos e mentais ${ }^{2}$.

Tal questão tornou-se ainda mais preocupante diante da situação causada pela coronavirus disease 2019 (Covid-19), pois os(as) estudantes ficaram ainda mais fragilizados(as). Por causa do isolamento social, recomendado pela maioria dos órgãos de saúde para minimizar a disseminação do novo coronavírus ${ }^{3}$, o sentimento de incerteza e a ausência das atividades diárias desencadearam tristeza, desânimo, ansiedade, apatia, angústia, entre outras sensações que formam um ambiente nocivo à saúde. Nesse contexto, a promoção da saúde tornou-se ainda mais fundamental, uma vez que visa capacitar/empoderar as pessoas a buscar melhor qualidade de vida, o que envolve a escolha de hábitos mais saudáveis. Para isso, é preciso ter acesso à informação e aos recursos necessários para que haja maior controle da própria saúde e cuidado com ela 4 .

Diante disso, a Associação Atlética Acadêmica Marcel Resende Davi (AAAMRD), criada em 1999 para representar a atividade desportiva universitária da Faculdade de Medicina da Universidade Federal de Uberlândia (UFU), guarda sua responsabilidade social de promoção da saúde física e mental de seus(as) estudantes. Em consonância com as Diretrizes Curriculares Nacionais (DCN) da graduação de Medicina ${ }^{5}$, a AAAMRD tem como função incentivar e promover a prática de esporte no meio acadêmico, conforme propaga o Plano Nacional de Educação Física e Prática de Desporto ${ }^{6}$. Dessa forma, percebe-se a importância dessa instituição para a melhor qualidade de vida dos(as) estudantes, o que impacta, inclusive, o processo de ensino-aprendizagem.

Por meio de treinos de diversas modalidades, coletivas e individuais, e da participação em eventos esportivos universitários, as associações atléticas acadêmicas (AAA) introduzem a prática desportiva e proporcionam importante integração, além de experiências significativas. Contudo, diante do contexto pandêmico, as ações mediadas pela atlética foram comprometidas, já que os treinos presenciais se tornaram impossibilitados. Mesmo com as dificuldades, valendo-se do comprometimento da instituição com os(as) estudantes que representa, a AAAMRD procurou desenvolver ações para promover a saúde dos(as) graduandos(as). Assim, formulou-se um desafio virtual entre as equipes desportivas da Medicina da UFU, incluindo todos(as) atletas, para que juntos(as) buscassem um modo de vida mais ativo que levasse em conta a importância da atividade física para a saúde integral do indivíduo e a redução da sua vulnerabilidade neste momento de pandemia.

Dessa forma, objetiva-se relatar a experiência da AAAMRD com este projeto, além de ressaltar a importância das AAA e das atividades esportivas como promotoras da saúde no âmbito acadêmico, especialmente no atual contexto de pandemia. Desse modo, esse desafio pode inspirar outras instituições a usufruir da tecnologia, em tempos de isolamento social, em prol do incentivo a hábitos saudáveis e, além disso, reforçar a relevância do esporte para o bem-estar geral do indivíduo.

Este relato de experiência enquadra-se no item VIII do artigo $1^{\circ}$ da Resolução n 510, de 7 de abril de 2016, do Conselho Nacional de Saúde, não necessitando, assim, de submissão ao Comitê de Ética em Pesquisa com Seres Humanos ${ }^{7}$.

\section{RELATO DE EXPERIÊNCIA}

A AAAMRD, atualmente constituída por 32 alunos da Faculdade de Medicina da UFU, elabora e organiza projetos esportivos a partir de reuniões semanais. Ademais, oferece treinos para 22 equipes, femininas e masculinas, de 16 modalidades esportivas: basquete, futsal, futebol de campo, handebol, peteca, vôlei, atletismo, judô, natação, eSports, jiujítsu, pebolim, sinuca, tênis de mesa, tênis de campos e xadrez.

Os treinamentos são abertos para todos(as) graduandos(as) em Medicina, incluindo tanto pessoas que sempre praticaram esportes quanto as que querem começar a praticá-los. Dentre os(as) 600 alunos(as), 156 participam ativamente das atividades esportivas promovidas pela AAAMRD e, portanto, são considerados(as) atletas. Os treinos objetivam não só promover saúde, como também preparar os times para competições universitárias.

Diante do contexto pandêmico, apesar da suspensão das atividades da UFU, a AAAMRD continua com suas reuniões semanais, ainda que remotamente, a fim de prosseguir com suas ações. Nesse sentido, um desafio envolvendo as equipes desportivas foi desenvolvido e executado virtualmente, por meio do aplicativo de conversas WhatsApp ${ }^{\circledR}$, objetivando incentivar os(as) estudantes a continuar realizando exercícios físicos e, consequentemente, cuidando da própria saúde física e mental, a fim de reduzir suas vulnerabilidades em tempos de pandemia.

O desafio consistiu em uma competição entre as equipes esportivas do curso de Medicina da UFU, as quais deveriam buscar a maior média de treinos físicos por atleta. Esse desafio ocorreu de forma remota, e todos(as) atletas foram 
estimulados(as) e aconselhados(as) a realizá-lo em casa. Assim, por meio do WhatsApp ${ }^{\circledR}$, no qual as equipes possuem grupos próprios com seus membros, os(as) atletas deveriam enviar fotos ou vídeos realizando atividades físicas.

Cada atleta poderia enviar um treino por dia e no máximo cinco treinos por semana, sem exigência de tempo mínimo ou máximo de atividade. Assim que enviasse a mídia, o(a) atleta atualizava uma lista no grupo com a quantidade de treinos que havia feito na semana proposta. As fotos e os vídeos ficaram restritos ao grupo das equipes por se tratar de um ambiente privado, fato que buscou atrair os(as) participantes que não gostam de se expor publicamente nas mídias digitais.

Desde o início da idealização da proposta, a atlética atentou-se à forma como esses treinos seriam realizados pelos(as) atletas. Assim, visando fornecer apoio para que as atividades físicas fossem realizadas de forma segura, de modo a evitar lesões e desgastes excessivos, fez-se uma parceria com profissionais capacitados para que, diariamente, a AAAMRD disponibilizasse em suas redes sociais opções de atividades físicas para realizar em casa, para todos(as) que se interessassem.

Como se trata de uma competição, a atlética buscou formas de incentivar os grupos e atletas a fim de obter maior adesão. Para isso, as três equipes com melhores desempenhos receberam premiação em dinheiro: $\mathrm{R} \$ 200,00, \mathrm{R} \$ 100,00$ e $\mathrm{R} \$ 50,00$. O resultado foi calculado a partir da média de número de treinos em relação ao número de atletas do time, visto que os times possuem números distintos de membros e uma simples soma dos treinos não seria justa. Os resultados preliminares da competição foram divulgados periodicamente nas redes sociais e nos grupos, como estratégia motivacional para a prática de exercícios físicos ${ }^{8}$.

Com o início do desafio, percebeu-se que, além dos treinos disponibilizados pela AAAMRD nas redes sociais, os(as) atletas usaram aplicativos específicos para atividades físicas, que contam com uma grande variedade de exercícios. Os(as) atletas compartilharam entre si esses programas, destacando vantagens e desvantagens de cada um, como forma de incentivar os(as) colegas. A fim de fazer com que as novas alternativas de exercícios alcançassem todos(as) participantes, a atlética disponibilizou em sua rede social os principais recursos utilizados.

Ao fim, o desafio teve duração de 11 semanas, contando com 14 equipes participantes e um total de 112 atletas. Das médias finais (Tabela 1), as três primeiras colocadas foram, respectivamente, vôlei feminino, natação e futsal feminino. Como forma de avaliar o desafio e compreender os pontos de aprimoramento para ações futuras, aplicou-se um questionário semiestruturado (Quadro 1) após o término do desafio - a apresentação desse instrumento de avaliação serviu para compreender a experiência, não se configurando como pesquisa. Além disso, foi importante para descrever a experiência por aqueles(as) que a vivenciaram. O questionário de preenchimento voluntário foi enviado aos grupos dos times, contando com 96 respostas.

Tabela 1. Número de atletas, número de treinos e média de treinos por equipe participante

\begin{tabular}{lccc}
\hline \multicolumn{1}{c}{$\begin{array}{c}\text { EQUIPES } \\
\text { PARTICIPANTES }\end{array}$} & $\begin{array}{c}\text { Número de } \\
\text { atletas }\end{array}$ & $\begin{array}{c}\text { Número de } \\
\text { treinos }\end{array}$ & MÉDIA \\
\hline Basquete masculino & 11 & 5 & 0,5 \\
Basquete feminino & 9 & 314 & 34,9 \\
Futsal masculino & 22 & 337 & 15,3 \\
Futsal feminino & 19 & 819 & 43,1 \\
Futebol de campo & 27 & 251 & 9,3 \\
Handebol masculino & 12 & 164 & 13,7 \\
Handebol feminino & 17 & 632 & 37,2 \\
Peteca & 13 & 324 & 24,9 \\
Vôlei masculino & 17 & 136 & 8,0 \\
Vôlei feminino & 13 & 684 & 52,6 \\
Atletismo & 28 & 532 & 19,0 \\
Natação & 21 & 916 & 43,6 \\
Tênis de mesa e de & 8 & 80 & 10,0 \\
campo & 11 & 435 & 39,5 \\
Judô e jiu-jítsu feminino & & & \\
\hline
\end{tabular}

Fonte: Elaborada pelos autores

Quadro 1.Questionário semiestruturado proposto para avaliação do desafio realizado

\section{Questionário Desafio Virtual AAAMRD}

Você está treinando durante a semana? ( ) SIM ( ) NÃO

Qual sua frequência de treinos?
( ) 1 vez por semana
( ) 2 vezes por semana
( ) 3 vezes por semana ( ) 4 vezes por semana
( ) 5 vezes por semana ( ) 6 vezes por semana
( ) 7 vezes por semana

Se não está treinando, qual o motivo?

Você realizou os exercícios disponibilizados pela AAAMRD nas redes sociais por algum período?

( ) SIM ( ) NÃO

Caso não tenha realizado, qual o motivo?

Deixe sua opinião sobre os treinos disponibilizados, mesmo que não tenha participado deles.

Você participou do desafio virtual esportivo entre os times? ( ) $\operatorname{SIM}($ ) NÃO

Deixe sua opinião sobre o desafio, mesmo que não tenha participado dele.

Caso não tenha participado, qual o motivo?

Espaço livre para críticas, elogios, opiniões ou ideias sobre o que podemos tentar fazer para envolver ainda mais nossos(as) atletas e contribuir para a atividade física durante esse cenário.

Fonte: Elaborado pelos autores 
A grande maioria afirmou que estava treinando, com frequência média de quatro a cinco treinos por semana. Entre aqueles(as) que não estavam se exercitando, observaramse justificativas como preguiça, falta de motivação e tristeza. Ademais, os(as) que utilizaram os treinos disponibilizados pela AAAMRD nas redes sociais parabenizaram a iniciativa. Alguns apontaram que os exercícios disponibilizados ficaram cansativos e desgastantes com a longa duração do desafio. $\mathrm{Na}$ área destinada para opinião a respeito do desafio, os elogios foram constantes, com comentários referentes: à importância do desafio na realização de treinos constantemente, com o estabelecimento de uma rotina diária; ao fato de a maior motivação ser ajudar o time; à relevância desse incentivo para manter-se ativo(a) e para a saúde física e mental; à criação de vínculo e à manutenção do contato entre os(as) atletas dos times, mesmo que a distância.

\section{Refletindo sobre a experiência: a responsabilidade social e a promoção da saúde}

O comprometimento da AAAMRD, referente à sua responsabilidade social sobre o incentivo ao esporte universitário, mostrou-se essencial na criação e execução do desafio, concretizando seu dever legal de representação esportiva ${ }^{6}$. Percebe-se isso pelo engajamento e pela preocupação em manter essa proposta, ainda que perante as adversidades provocadas pela pandemia. Esta provocou alterações significativas na rotina da população, sobretudo pelo distanciamento, o qual gerou maior vulnerabilidade individual, fragilizando sentimentos e emoções, e, consequentemente, o bem-estar físico e mental das pessoas, de forma a torná-las expostas ao adoecimento ${ }^{9}$.

Os(as) estudantes, em especial, ao terem seu cronograma acadêmico e rotina interrompidos, apresentam dificuldade de se manter motivados(as) e tendem à frustração ante a sensação de improdutividade. Dessa forma, a retomada de parte do cotidiano dos(as) atletas, mediante a constância na prática de exercícios físicos, contribui para a redução dos eventuais transtornos que podem afetar a vida deles(as) nesse contexto.

Essa estratégia mostra-se relevante porque os exercícios físicos consistem em recursos não farmacológicos de promoção da saúde que contribuem para a prevenção de doenças metabólicas, imunológicas e psicológicas, além de serem fundamentais na manutenção da capacidade funcional dos indivíduos, auxiliando no cuidado integral da saúde e no estilo de vida ativo ${ }^{10}$. Desse modo, o estímulo a essa prática possibilita significativos impactos na saúde dos(as) estudantes de Medicina, especialmente no aspecto mental, crucial para a redução do estresse e da ansiedade, exacerbados pelo contexto de pandemia9.

Nesse sentido, vale ressaltar que exercícios aeróbicos impactam direta e indiretamente a saúde cerebral dos indivíduos, como mecanismo terapêutico de cuidado da saúde mental ${ }^{11,12}$. Ademais, há liberação de endorfina como consequência da prática de exercícios físicos que possibilita sensação de euforia, tranquilidade, bem-estar e redução de ansiedade ${ }^{13}$, permitindo equilíbrio psicoemocional, que ameniza impactos negativos do meio externo. Assim, o estresse, que afeta biológica, psicológica e socialmente o indivíduo, pode ser amenizado com essa prática, promovendo significativa relação de compatibilidade entre saúde física e mental.

Diante disso, buscou-se estipular a constância nos treinos em prol da manutenção de parte da rotina. Em contrapartida, a atlética atentou-se ao fato de que esse compromisso diário poderia tornar-se desgastante, física e mentalmente, diante da cobrança e do compromisso com o time. Dessa forma, seguindo as orientações da Organização Mundial da Saúde (OMS), que recomenda 150 minutos semanais de atividades físicas de leve a moderada intensidade ou 75 minutos de alta intensidade ${ }^{14}$, optou-se por limitar a cinco treinos por semana e máximo de um treino por dia. Por isso, ficaram a critério do(a) atleta a duração e a modalidade de exercício físico a ser realizado, respeitando os limites de cada um(a).

Em meio à pandemia, academias de ginástica, clubes e ginásios de práticas esportivas foram fechados, o que reduziu as possibilidades estruturais de manutenção do estilo de vida ativo e dos treinos proporcionados pela AAAMRD, até então majoritariamente coletivos e presenciais. Entretanto, essas dificuldades foram amenizadas pelo impacto da tecnologia ${ }^{15}$, já que o progresso tecnológico permitiu que o desafio fosse criado e aplicado virtualmente no WhatsApp ${ }^{\circledR}$, sem que os(as) participantes precisassem sair de casa.

Tendo em vista a realização do desafio dentro desses grupos, um aspecto fundamental para o sucesso da ação foi o espírito de coletividade surgido dentro dos times. Motivados a ganhar, os(as) atletas se uniram em prol de um objetivo maior, para além de sua individualidade. É evidente que os benefícios individuais, como bem-estar físico e mental, além da manutenção de um bom condicionamento físico, não foram desconsiderados. Contudo, foi notório que essa competitividade amistosa, a qual objetiva sucesso não em detrimento do outro, mas a partir da força do próprio grupo, fez com que a maior motivação fosse advinda do desejo de ajudar o time a alcançar o pódio.

Diante disso, eram constantes as mensagens de incentivo, de congratulações e até de "cobrança" nos grupos virtuais. Ademais, os(as) atletas compartilhavam entre si aplicativos, lives, vídeos e séries de treinos seguros e interessantes que realizavam. Essa troca de vivências permitiu novas experiências, por possibilitar o contato com vários tipos de exercício físico, que 
variavam de yoga, alongamentos e dança a exercícios aeróbicos de alta intensidade, o que fortaleceu o trabalho em equipe ${ }^{15}$.

Essa interação entre os(as) atletas do time contribuiu não só para a constância de treinos, mas também para uma interação diária. A partir disso, foi possível perceber o surgimento de uma rede de apoio social entre os(as) estudantes, caracterizada pela continuidade e/ou pelo surgimento de vínculos significativos e afetivos de apoio e proteção ${ }^{16}$. Assim, os(as) estudantes trocavam informações para além de exercícios físicos, com conversas acerca de suas rotinas, expectativas futuras, medos, inseguranças e saudades.

Tal fato mostrou-se extremamente relevante, uma vez que grande parte dos(as) universitários(as) estão acostumados(as) com uma significativa interatividade social, tendo em vista que, cotidianamente, relacionam-se com indivíduos que praticam atividades comuns às suas, expandindo suas experiências ${ }^{17}$. Os(as) atletas, em especial, vivenciam uma grande diversidade de interações sociais, já que em treinos e competições convivem com muitas pessoas, de diversos períodos do curso. Essa troca de experiências expressa-se de forma semelhante à que ocorre no projeto de mentoring, fundamental para a formação pessoal e profissional do indivíduo, uma vez que amplia sua rede de relações ao colocá-lo em contato com acadêmicos(as) dos períodos tanto iniciais como finais da graduação ${ }^{18}$.

Outro aspecto relevante foi a escolha da premiação em dinheiro, que buscou incitar ainda mais o espírito de coletividade dentro das equipes. Assim, ficou a critério de cada uma como usufruir desse prêmio, de acordo com suas distintas necessidades e motivações. Dentre as opções mais propostas, podem-se citar: compra de equipamentos esportivos, criação de novos uniformes ou blusas de passeio e realização de confraternizações. Nota-se, portanto, que a coletividade prevaleceu em detrimento das vontades individuais, mesmo no momento de usufruir da premiação.

Ao final do desafio, aplicaram-se formulários de feedback com o intuito de avaliar a adesão, importância e efetividade dessa ação, pois essa ferramenta permite uma análise da performance da atividade, sendo essencial para mensurar até que ponto os objetivos foram alcançados. Dessa forma, contribui para o aprimoramento da organização e dos(as) colaboradores(as), servindo também como instrumento motivacional para realização de novas ações ${ }^{19}$.

A partir disso, reconheceram-se algumas limitações da ação, como: cerca de $28 \%$ dos(as) atletas não aderiram ao desafio; nem todos(as) os(as) estudantes tiveram a oportunidade de participar, já que a ação foi restrita aos(às) atletas; as diferenças de coesão das equipes e da integração entre os(as) atletas prejudicaram a avaliação da média do número de treinos, diante das diferentes motivações dos times; a duração longa e não predeterminada do desafio gerou desgaste e cansaço, desmotivando os(as) atletas nas semanas finais. Dessa forma, ressalta-se a importância de analisar esses aspectos, visando aprimorá-los para as próximas ações.

Em contrapartida, os pontos positivos foram expressivos, superando as eventuais limitações do desafio virtual. Ressaltase a importância: do desafio na prática e constância de exercícios físicos; da coletividade na motivação dos(as) atletas; da privacidade e do ambiente acolhedor garantidos pelos grupos de WhatsApp ${ }^{\circledR}$; e da perpetuação de parte do contato e vínculo entre os(as) atletas da equipe, mesmo diante do distanciamento social. Por fim, nota-se a efetividade do desafio, promovido pela AAAMRD, na promoção da saúde física e mental dos(as) estudantes, cumprindo, dessa forma, a sua responsabilidade social ${ }^{6}$.

Ademais, ressalta-se que, em consonância com as DCN do curso de graduação em Medicina, a AAAMRD contemplou todas as áreas propostas: atenção, gestão e educação em saúde. O primeiro aspecto relacionou-se à preocupação com a saúde integral dos(as) atletas mediante construção do desafio para autocuidado. Sobre a gestão em saúde, nota-se que a atlética soube utilizar a tecnologia como aliada e aproveitou sua posição de instituição acadêmica para fortalecer seu comprometimento social. Além disso, nota-se a contribuição da AAAMRD para a educação médica, a qual preza o desenvolvimento de noções de responsabilidade, habilidades comunicativas, empatia e trabalho em equipe, fundamentais para a formação pessoal e profissional dos(as) estudantes e atuação na prática médica ${ }^{5}$.

\section{CONCLUSÃO}

Ante o cenário atual, é notório que a pandemia da Covid-19 propiciou prejuízos à saúde dos indivíduos, especialmente dos(as) universitários(as) ${ }^{9}$, evidenciando a necessidade de reorganização das atividades físicas diante do isolamento social. Vale lembrar-se da vulnerabilidade já existente desses(as) estudantes, em razão do desgaste físico e mental, fruto da extensa carga horária que compõe a graduação. Assim, percebe-se a importância de promover a saúde, principalmente em situações de fragilidade social como essa, a fim de conscientizar as pessoas a adotar um estilo de vida mais saudável e ativo.

Nessa perspectiva, constata-se a relevância da atuação da AAAMRD, ao evidenciar a associação entre a atividade física e a saúde integral dos indivíduos. Desse modo, ao admitir a sua responsabilidade social, a instituição corrobora o seu papel na educação em saúde à medida que contribui para a formação de valores pessoais dos(as) seus(suas) estudantes, conforme fundamentam as $\mathrm{DCN}^{5}$. Ademais, 
essa atuação também ratifica o papel mediador da atlética no contato dos(as) graduandos(as) com a prática esportiva, fornecendo informações e recursos necessários para que sejam protagonistas do processo de autocuidado em saúde, conforme preconizado pela Carta de Ottawa4.

Evidencia-se, portanto, que a ação de promoção da saúde realizada pela $A A A M R D$ alcançou seu objetivo inicial de incentivar a prática esportiva e o exercício físico dentro das limitações impostas pelo isolamento social, haja vista a grande quantidade de atletas que participaram e o retorno positivo destes(destas) para a instituição. Espera-se que com este projeto a atlética seja exemplo para outras instituições estudantis e possa disseminar essa ideia. Por fim, houve a possibilidade de analisar os pontos positivos e negativos do desafio, o que contribui para um melhor planejamento das próximas ações, tendo em vista a pretensão da instituição de alcançar mais pessoas na promoção da saúde.

\section{AGRADECIMENTOS}

Agradecemos primeiramente à Universidade Federal de Uberlândia (UFU), em especial à Faculdade de Medicina e a todo seu corpo docente, discente e técnico-administrativo, que sempre apoiaram e incentivaram a Associação Atlética Acadêmica Marcel Resende Davi (AAAMRD) em todos os seus projetos que visam à promoção de saúde dos alunos de Medicina. Agradecemos também a todos(as) os(as) integrantes da AAAMRD que nos ajudaram na construção e realização do projeto em questão e aos(às) atletas e aos demais participantes, sem os(as) quais nada seria possível. Por fim, agradecemos ao orientador do manuscrito, Prof. Gustavo Antonio Raimondi, que nos auxiliou tão bem na execução do presente relato de experiência.

\section{CONTRIBUIÇÃO DOS(AS) AUTORES(AS)}

Todos(as) os(as) autores(as) participaram ativamente de todas as etapas de elaboração do manuscrito.

\section{CONFLITO DE INTERESSES}

Os(as) autores(as) declaram não haver conflito de interesses.

\section{FINANCIAMENTO}

Declaramos que não houve financiamento para a realização desta pesquisa.

\section{REFERÊNCIAS}

1. Feodrippe ALO, Brandão MCF, Valente TCO. Qualidade de vida de estudantes de Medicina: uma revisão. Rev Bras Educ Med. 2013;37(3):418-28.

2. Carmo ME, Guizardi FL. O conceito de vulnerabilidade e seus sentidos para as políticas públicas de saúde e assistência social. Cad Saude Publica. 2018;34(3):e00101417.

3. Islam N, Sharp SJ, Chowell G, Shabnam S, Kawachi I, Lacey B, et al. Physical distancing interventions and incidence of coronavirus disease 2019: natural experiment in 149 countries. BMJ. 2020;370:m2743.

4. Brasil. As cartas da promoção da saúde. Brasília: Ministério da Saúde; 2002.

5. Brasil. Resolução no 3, de 20 de junho de 2014. Institui Diretrizes Curriculares Nacionais do Curso de Graduação em Medicina e dá outras providências. Diário Oficial da União, Brasília; 23 jun 2014. Seção 1, p. 8-11.

6. Starepravo FA, Reis LJA, Mezzadri FM, Marchi Júnior W. Esporte universitário brasileiro: uma leitura a partir de suas relações com o Estado. Rev Bras Ciênc Esporte. 2010;31(3)131-48.

7. Brasil. Resolução n 510 , de 7 de abril de 2016. Dispõe sobre as normas aplicáveis a pesquisas em Ciências Humanas e Sociais. Diário Oficial da União, Brasília; 24 maio 2016. Seção 1.

8. Cechin FM, Evangelista PHM, Saldanha RP, Balbinotti MAA, Klering RT, Barbosa MLL, et al. Motivação competitiva de "squashistas" juvenis federados. Rev Bras Educ Fís Esp. 2014;28(3):469-80.

9. Maia BR, Dias PC. Ansiedade, depressão e estresse em estudantes universitários: o impacto da COVID-19. Estud Psicol. (Campinas). 2020;37:e200067.

10. Raiol RA. Praticar exercícios físicos é fundamental para a saúde física e mental durante a pandemia da Covid-19. Braz J Health Rev. 2020;3(2):2804-13.

11. Vorkapic-Ferreira C, Góis RS, Gomes LP, Britto A, Afrânio B, Dantas EHM. Nascidos para correr: a importância do exercício para a saúde do cérebro. Rev Bras Med Esporte. 2017;23(6):495-503.

12. Campos CG, Muniz LA, Belo VS, Romano MCC, Lima MC. Conhecimento de adolescentes acerca dos benefícios do exercício físico para a saúde mental. Cien Saude Colet. 2019;24(8):2951-8.

13. Werneck FZ, Bara Filho MG, Ribeiro LCS. Mecanismos de melhoria do humor após o exercício: revisitando a hipótese das endorfinas. Revista Brasileira de Ciência \& Movimento. 2005;13(2):135-44.

14. World Health Organization. Stay physically active during self-quarantine. WHO; 2020 [acesso em 21 jul 2020]. Disponível em: https://www.euro. who.int/en/health-topics/health-emergencies/coronavirus-covid-19/ technical-guidance/stay-physically-active-during-self-quarantine.

15. Chaves PLL, Fernandes Neto P. Integração de tecnologias para promoção da saúde mental da comunidade acadêmica [trabalho de conclusão de curso]. Mossoró: Universidade Federal Rural do Semi-Árido; 2019.

16. Juliano MCC, Yunes MAM. Reflexões sobre rede de apoio social como mecanismo de proteção e promoção de resiliência. Ambient Soc 2014;17(3):135-54.

17. Davis C, Silva MASS, Esposito YL. Papel e valor das interações sociais em sala de aula. Cad Pesqui. 1989;(71):49-54.

18. Martins AF, Bellodi PL. Mentoring: uma vivência de humanização e desenvolvimento no curso médico. Interface Comun Saúde Educ. 2016;20(58):715-26.

19. Macedo EL. A importância do feedback no processo de avaliação de desempenho [trabalho de conclusão de curso]. Leme: Centro Universitário Anhanguera de Leme; 2019. 